\title{
DE LA FONÉTICA DEL HABLA ESPONTÁNEA A LA FONOLOGÍA DE LA COMPLEJIDAD
}

\author{
FROM PHONETICS OF SPONTANEOUS SPEECH TO PHONOLOGY OF COMPLEXITY \\ FRANCISCO JOSÉ CANTERO SERENA \\ Laboratori de Fonètica Aplicada-Universitat de Barcelona
}

\section{RESUMEN:}

El análisis fonético del habla espontánea nos enfrenta a numerosos interrogantes sobre la naturaleza de los sonidos del habla, así como sobre su interpretación fonológica. En este artículo revisamos algunos de esos interrogantes, así como sus implicaciones teóricas y prácticas, y proponemos un enfoque alternativo, basado en la teoría de la competencia comunicativa y en los principios del pensamiento complejo.

PALABRAS CLAVE: Fonética, fonología, competencia comunicativa, pensamiento complejo, análisis acústico, habla espontánea, corpus orales.

\section{ABSTRACT:}

Phonetic analysis of spontaneous speech confronts us with numerous questions about the nature of speech sounds as well as their phonological interpretation. This article reviews some of these questions as well as their theoretical and practical implications, and proposes an alternative approach based on the theory of Communicative Competence and the principles of Complex Thought.

KeYwords: Phonetics, Phonology, Communicative Competence, Complex Thought, Acoustics Analysis, Spontaneous Speech, Oral Corpus. 


\section{FONÉTICA DE LO IMPREVISTO}

Podemos decir que el principio de la ciencia clásica es: legislar

(E. Morin, 2004)

La fonética ha ofrecido, tradicionalmente, modelos pronunciación muy cerrados, con una finalidad prescriptiva; modelos de pronunciación culta, correcta y eficaz, que permitían ofrecer referentes claros a los aprendices de un idioma, ya fueran nativos o extranjeros, y que venían a ser el correlato «en voz alta» de la norma escrita: las normas de pronunciación.

Sin abandonar del todo ese espíritu normativo, a lo largo del siglo veinte la fonética se ha ido adaptando a los métodos científicos (y pseudocientíficos ${ }^{1}$ ) de la moderna lingüística $\mathrm{y}$, en la actualidad, la entendemos como una disciplina esencialmente «experimental» (aunque, a veces, se emplee «experimental» más bien como sinónimo de «instrumental») ${ }^{2}$.

En realidad, la fonética siempre ha sido una disciplina empírica (instrumental) y los fonetistas han sido, con mucho, los primeros lingüistas de laboratorio: de hecho, ha sido precisamente en los laboratorios de fonética donde se han generado las normas de pronunciación correcta (sancionadas luego por las instituciones). Ejemplos muy claros son el manual de pronunciación de Rousselot para el francés (Rousselot y Laclotte, 1902) o el de Navarro Tomás para el español (Navarro Tomás, 1918), ambos realizados a partir del análisis instrumental de registros de voz.

Simultáneamente, la fonología, como disciplina teórica que ofrece una interpretación y/o una explicación del funcionamiento lingüístico de los sonidos del habla, fue relegando a la fonética como mera ciencia auxiliar y haciendo suyos los modelos de pronunciación que esta había elaborado. Entonces, los modelos de pronunciación tradicionales, que describían la pronunciación conveniente o deseable, la norma culta, se convirtieron en «sistemas fonológicos» que agotaban las posibilidades fonéticas del hablante (hasta el extremo de considerar «agramatical» buena parte de las pronunciaciones más comunes, como sabemos ahora).

En español, por ejemplo, Alarcos Llorach explica, en su Fonología española, que va a intentar «una descripción fonológica del español actual [...] el mismo estilo de español estudiado fonéticamente por Navarro Tomás» (1950: 143); Navarro Tomás, sin embargo, en su manual de pronunciación precisaba: «esta pronunciaciónpues, castellana sin vulgarismo y culta sin afectación, estudiada especialmente en el ambiente universitario madrileño, es la que en el presente libro se pretende describir», y añadía: «llamámosla correcta sin otro objeto que el de distinguirla de la pronunciación vulgar» (Navarro Tomás, 1918: 8).

Así pues, donde la fonética tradicional describía objetivamente un estilo determinado de habla (considerado mejor o más deseable) para establecer una norma social de pronunciación, la fonología ha postuladolas unidades y el funcionamiento del sistema de la

\footnotetext{
${ }^{1}$ Según la terminología de M. Bunge: $c f r$. Bunge, 1969 y, sobre todo, Bunge, 1983.

${ }^{2}$ Una confusión de términos que encontramos ya en la obra fundacional de Rousselot (1908).
} 
lengua (dejando aparte el estudio de las variantes geográficas y despreciando las variantes coyunturales).

Buena parte de la moderna fonética experimental sigue basándose en habla de laboratorio (muestras rara vez dialógicas, grabadas en condiciones no comunicativas, a menudo frases preparadas y leídas... incluso por el propio investigador) y persigue la comprobación de los detalles y las contradicciones teóricas de tal o cual tendencia teorética. Muy lejos, pues, de la descripción objetiva del habla genuina de los hablantes reales en situaciones auténticas de comunicación.

De hecho, el análisis del habla espontánea no siempre ha sido visto con buenos ojos por los autores más relevantes del campo, justamente porque lo que se perseguía era la comprobación de hipótesis (teóricas) y en habla espontánea no pueden controlarse todas las variables; solo muy recientemente el análisis del habla espontánea se ha convertido en un campo de estudio común entre los fonetistas.

Sin embargo, ocurre que las proyecciones teóricas de esta «fonética de las certezas» están llenas de zonas borrosas ${ }^{3}$ que llevan a una aplicabilidad muy discutible de los conocimientos generados por nuestra ciencia: zonas borrosas que implican, necesariamente, aplicaciones borrosas.

\subsection{Zonas borrosas, aplicaciones borrosas}

Las fonologías tradicionales, de corte estructuralista, a menudo confunden, como hemos visto, la descripción fonética con la prescripción (donde la norma es fonética y fonológica a la vez) y la prescripción con el «sistema». La investigación fonética, por tanto, suele plantearse a partir de apriorismos normativos.

También las fonologías alternativas a la tradición estructural, de tipo generativista, «no lineales» ${ }^{4}$, etc., siguen ancladas en la prescripción, rehúyen la descripción del habla espontánea (que, por norma general, no sigue las normas y, por tanto, se considera «agramatical») y sustituyen el análisis por la «representación», la proyección de reglas (donde las «reglas» vienen a ser «normas») o el mero etiquetaje.

Ningún sistema fonológico explica la realidad del habla, y su proyección en los diversos ámbitos de aplicación de la disciplina es, a menudo, ineficaz o incluso contraproducente:

En las aplicaciones didácticas de la fonética y la fonología, por ejemplo, suelen ofrecerse modelos unívocos y cerrados, con pocas variantes aceptables, a menudo

${ }^{3}$ Término con el que nos referimos a fenómenos abiertos o contradictorios como el yeísmo, el ceceo, los márgenes de dispersión solapados, etc., que no permiten cerrar nunca del todo los sistemas fonológicos. En español, por ejemplo, las variantes fonéticas del fonema /j/ van desde la casi vocálica [j] (como en mayo) a pronunciaciones prácticamente africadas [§j] (como en cónyuge): dos clases de sonidos en una única unidad fonológica ( $c f r$. Cantero, 2014a).

${ }^{4}$ En la fonología generativa clásica, la representación fonológica supone una disposición «lineal», secuenciada, de los segmentos en un único nivel, mientras que en las fonologías «no lineales» (como la fonología autosegmental o la fonología métrica) la representación fonológica se concibe en distintos niveles, en los que los rasgos se organizan jerárquicamente. Como veremos, la «no linealidad» de las modernas fonologías generativistas no debe confundirse con el concepto de «no linealidad» del pensamiento complejo, al que nos referiremos más adelante. 
centrados exclusivamente en la producción o la percepción de sonidos aislados o, a lo sumo, de sonidos en contacto: no se distinguen, en ningún caso, la producción, la percepción y la interacción como actividades comunicativas claramente diferenciadas ${ }^{5}$.

En general, la corrección fonética no es demasiado eficaz ${ }^{6}$ y pocas veces va más allá de ser un ejercicio pobre, centrado en el desarrollo de habilidades exclusivamente escolares: la percepción de pares mínimos y la pronunciación correcta de sonidos aislados, en efecto, poco tienen que ver con las habilidades comunicativas que se exigen a un hablante competente (que rara vez pronunciará un sonido aislado, y rara vez se enfrentará, a lo largo de su vida, a pares léxicos mínimos y descontextualizados como casa/caza o pollo/poyo).

En las demás aplicaciones de la fonética y la fonología (aplicaciones clínicas, forenses e industriales), estas disciplinas ofrecen, igualmente, modelos teóricos de referencia más pensados como norma que como descripción:

- en fonética clínica, poco tienen que ver las producciones reales de los pacientes (de una gran variabilidad) con los modelos teóricos de la lengua estándar;

- en fonética forense, las grabaciones dubitadas suelen ser de habla espontánea, poco explorada, mientras que las grabaciones indubitadas son de habla de laboratorio, a menudo leída (cuyo modelo de referencia, de nuevo, es la lengua estándar);

- en síntesis de voz, la naturalidad está reñida con los sistemas unívocos, por lo que a menudo se emplean unidades más creativas que las unidades fonológicas (como los difonemas ${ }^{7}$, etc.);

- en reconocimiento automático del habla, los modelos perceptivos de que se disponen solo permiten la percepción de palabras aisladas, pero no la percepción de habla continua.

La teoría, la norma y la realidad son contradictorias demasiadas veces, por lo que se hace necesario poder ofrecer modelos de pronunciación más realistas, más cercanos al habla real, basados en el análisis de los procesos comunicativos auténticos: el análisis del habla espontánea.

${ }^{5}$ El Marco común europeo de referencia para las lenguas (MCER) distingue cuatro actividades comunicativas diferenciadas: producción, percepción, interacción y mediación (Council for Cultural Cooperation, 2001).

${ }^{6}$ Nosotros preferimos un enfoque comunicativo de la corrección fonética: la «didáctica de la pronunciación» (cfr. Bartolí, 2005, 2012, 2014; Cantero, 1998, 2003; Giralt, 2012, 2014).

${ }^{7}$ Cfr. Guaus et al. (1997). 


\subsection{Análisis del habla espontánea}

A pesar de ser una actividad de investigación que despierta un creciente interés entre los fonetistas, aún no contamos con descripciones completas del habla espontánea que permitan ofrecer modelos teóricos alternativos a los sistemas fonológicos clásicos (basados en unidades unívocas o en reglas).

En nuestro centro de investigación ${ }^{8}$ tomamos el análisis del habla espontánea como nuestro objetivo prioritario, y a lo largo de los últimos años hemos realizado diversas investigaciones concretas que abarcan el análisis de la entonación del español, del catalán y del español hablado por extranjeros; del vocalismo del castellano; del vocalismo del catalán; y distintos aspectos del consonantismo del español ${ }^{9}$.

Los trabajos realizados hasta el momento nos aportan algunos resultados fragmentarios, de mucho interés, y nos provocan numerosas perplejidades teóricas.

Contamos con un amplio corpus de habla espontáne ${ }^{10}$ en español peninsular, con más de 600 informantes anónimos, procedentes de 10 variedades del español (Andalucía, Extremadura, Murcia, Castilla La Mancha, Castilla León, Madrid, Navarra, País Vasco, Asturias y Canarias) y está constituido por más de 3000 enunciados, todos ellos emitidos en situación de conversación genuina, no controlada, y con una excelente calidad de audio ( $c f r$. Ballesteros et al., 2010).

Podemos resumir algunos de los resultados de nuestros análisis en tres grandes apartados: vocalismo, consonantismo y prosodia.

Vocalismo: tanto en castellano (cfr. Alfonso, 2010, 2014) como en catalán ( $c f r$. Rius-Escudé, 2011, 2014; Rius-Escudé y Torras, 2014, 2015) observamos márgenes de dispersión solapados entre todas las vocales contiguas: /i/-/e/, /e/-/ $/$ /, / / /-/a/, /a/-/o/, /o//o/, /o/-/u/; pero también entre las vocales medias: /e/-/ع/-/o/-/o/. En pruebas perceptivas, este solapamiento es aún más notorio. En castellano, además, hemos comprobado que los diptongos constituyen una única unidad perceptiva ( $c f r$. Alfonso, 2010).

En habla espontánea, por tanto, el vocalismo no parece funcionar igual en producción que en percepción, y los márgenes de seguridad de sus unidades son, digámoslo así, más bien «inseguros».

Consonantismo: nos encontramos con numerosas realizaciones no esperables, referidas a las laterales ( $c f r$. Andrés Edo, 2014), las vibrantes ( $c f r$. Ortiz de Pinedo 2012, 2014) y las aproximantes del español (cfr. Sola Prado 2011, 2014a, 2014b).

Hemos visto que, por ejemplo, las realizacines de /1/ desaparecen o se funden con la vocal contigua en un $25 \%$ de las ocasiones, en posición de semimargen silábico; en un $16 \%$, en posición implosiva; y en un $10 \%$, en posición intervocálica.

${ }^{8}$ El Laboratori de Fonètica Aplicada de la Universitat de Barcelona, donde trabaja el Grup de

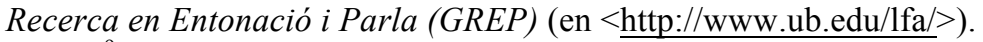

${ }^{9} \mathrm{Vid}$. una visión de conjunto de los distintos trabajos que se llevan a cabo, en el volumen 9-10 de

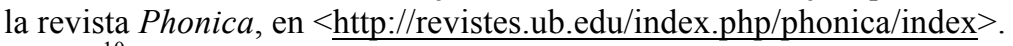

${ }^{10}$ El corpus LFA, consultable desde finales de 2015 en nuestra web (vid. supra), incluye diversos corpus de español peninsular y de catalán, en una primera fase; paulatinamente, iremos incluyendo los corpus de español hablado por taiwaneses, brasileños, húngaros, suecos, italianos... y nuestros corpus de habla infantil. 
Con respecto a las aproximantes $[\beta, \delta, \gamma]$, también es frecuente que desaparezcan o que se fundan con las vocales contiguas, en todas las posiciones analizadas; en cambio, en numerosas ocasiones, y contra pronóstico, se realizan como obstruyentes.

Las vibrantes, por su parte, tienden a realizarse masivamente como aproximantes: en un $71 \%$ de las ocasiones, en posición intervocálica; y en un $50 \%$, en posición inicial.

En conjunto, observamos que más de un $70 \%$ de las realizaciones de estas consonantes en habla espontánea son realizaciones no esperables, no previstas por los modelos fonológicos ni por los modelos fonéticos de referencia.

En habla espontánea, por tanto, el consonantismo parece contradecir la mayoría de las restricciones que le imponen los modelos teóricos.

Prosodia: el análisis de la entonación es un buen ejemplo de cómo los sistemas clásicos de análisis fonético y fonológico carecen de herramientas precisas para afrontar (describir, entender, explicar y aplicar) los fenómenos del habla espontánea.

En las curvas melódicas encontramos muy pocos rasgos analizables, todos ellos cifrados en la frecuencia fundamental de los sonidos que componen el discurso: tonos que ascienden o descienden y que constituyen una única melodía. Esa melodía, sin embargo, no es el significante de un único significado, sino que es el portador de múltiples valores. Podríamos llamar a este fenómeno: «sincretismo melódico».

La inflexión final de un contorno ${ }^{11}$, por ejemplo, informa del valor lingüístico de ese contorno (es decir, de si se trata de una entonación neutra, interrogativa, etc.); pero también, y a la vez, informa de la variedad dialectal del hablante; y también, simultáneamente, informa del valor de cortesía que el hablante está dando al enunciado, de su carga emocional y/o de su focalización ${ }^{12}$. Los tres niveles de análisis ${ }^{13}$ ocurren en el mismo rasgo, en la misma inflexión tonal.

Estesincretismo melódico suele confundir a los investigadores, que prefieren realizar sus análisis obviándolo, por ejemplo analizando únicamente frases leídas y descontextualizadas, en las que se ha controlado la estructura sintáctica de las mismas.

En habla espontánea, por tanto, la entonación se resiste a los meros etiquetados lingüísticos, y cada rasgo aporta información múltiple que no ha sido codificada en los modelos teóricos de las fonologías clásicas.

Todos estos fenómenos provocan una perplejidad teórica que solo podemos neutralizar si analizamos habla de laboratorio (es decir, si damos la espalda a la comunicación auténtica). Pero la realidad es muy tozuda y nuestra ciencia muy curiosa (especialmente, desde que contamos con métodos robustos de análisis acústico y melódico).

${ }^{11} \mathrm{O}$ el «núcleo», o el «tonema», según la escuela de análisis.

12 Sobre la cortesía en la entonación del español: $c f r$. Devís (2011); del catalán: $c f r$. Devís y Cantero (2014); sobre la entonación de foco: cfr. Cantero et al. (2005) y Font-Rotchés (2011), (2014); sobre la entonación emocional: $c f r$. Cantero (2014b).

${ }^{13}$ Que en nuestro modelo de «análisis melódico del habla» llamamos entonación prelingüística, lingüística y paralingüística (cfr. Cantero, 2002; Cantero y Mateo, 2011). 


\subsection{La pronunciación no es unívoca}

La producción, la percepción, la mediación y la interacción constituyen actividades comunicativas diferenciadas, que implican competencias fónicas específicas, que bien podrían constituir sistemas fonéticos diferenciados, y cuya interpretación fonológica debería ser igualmente diferenciada.

La pronunciación no es unívoca: participan en ella múltiples códigos orales (dialectales, de registro, de estilo, etc.: cfr. Cantero, 2004) que, a su vez, son códigos multisistémicos: verbales y no verbales, simultáneamente (cfr. Torregrosa, 2006, 2011, 2014). Un mismo hablante emplea códigos alternativos en función del contexto, de la situación comunicativa, de su rol en el intercambio comunicativo, entre otros factores, sin dejar de hablar (ni de sentir que habla) el mismo idioma. Por ejemplo, es frecuente el cambio de código fonético ${ }^{14}$ para enfatizar o focalizar una parte del enunciado.

Cuando analizamos el habla espontánea, esta multiplicidad de la pronunciación es incontestable, pero hasta ahora no había sido contemplada con seriedad por las teorías tradicionales, que habían relegado el fenómeno al cajón de sastre de la «fonoestilística» ${ }^{15}$ y la dialectología.

En Cantero (2014a) hemos ensayado una primera aproximación a las características de estos sistemas fónicos diferenciados, que en los modelos clásicos no acababan de encajar, pero que desde una nueva perspectiva, basada en la moderna concepción de la Competencia Comunicativa, adquieren pleno sentido.

\section{UNA PERSPECTIVA ALTERNATIVA: LA COMPETENCIA COMUNICATIVA}

Partimos deuna visión de conjunto de la competencia comunicativa, desde una perspectiva en la que se comprenden las diversas actividades comunicativas (competencias específicas), los saberes y recursos que permiten afrontarlas (competencias estratégicas), así como la complejidad de códigos que maneja el hablante (la estrella comunicativa) ${ }^{16}$.

\footnotetext{
${ }^{14}$ El cambio de código o codeswitching suele referirse al empleo ocasional de algún elemento de un idioma en un discurso codificado en otro idioma, como usar una expresión en inglés cuando se está hablando en español ( $c f r$. Gumperz, 1982; Heller, 1988). Entendemos que también es común, en un mismo idioma, introducir en el discurso elementos de códigos fónicos alternativos, como por ejemplo modificar el timbre vocálico para remedar la pronunciación de otra persona, o imitar un acento, etc. Véase una visión del fenómeno en Cantero y de Arriba (1996).

${ }^{15}$ Curiosamente, la «estilística» lingüística fue propuesta por Charles Bally, unos de los editores del Curso de lingüística general (de Ferdinand de Saussure), obra fundacional del estructuralismo lingüístico, cuyas derivaciones teóricas constituyen la base de todas las fonologías actuales. Pocos autores han seguido una línea «estilística», entre los cuales tal vez el más solvente sería Fónagy (1983).

${ }^{16}$ Nuestro modelo de Competencia Comunicativa está explicado en Cantero (2008) y adaptado al ámbito de la fonética en Cantero (2014a).
} 


\subsection{Competencias especificas y estratégicas}

Las competencias específicas son los recursos que permiten al hablante gestionar sus actividades comunicativas:

- la competencia productiva es la capacidad de generar discursos inteligibles, de producir lenguaje monológico,

- la competencia perceptiva es la capacidad de comprender discursos ajenos,

- la competencia mediadora es la capacidad de hacer comprender discursos ajenos (como parafrasear, resumir, adaptar, traducir, etc. $)^{17}$,

- la competencia interactiva es la capacidad de gestionar la comunicación con otros interlocutores.

Estas competencias se relacionan entre sí con una cierta jerarquía, por cuanto el centro de todas ellas es la competencia interactiva, es decir, la conversación como eje de la comunicación humana. Las representamos así (figura 1):

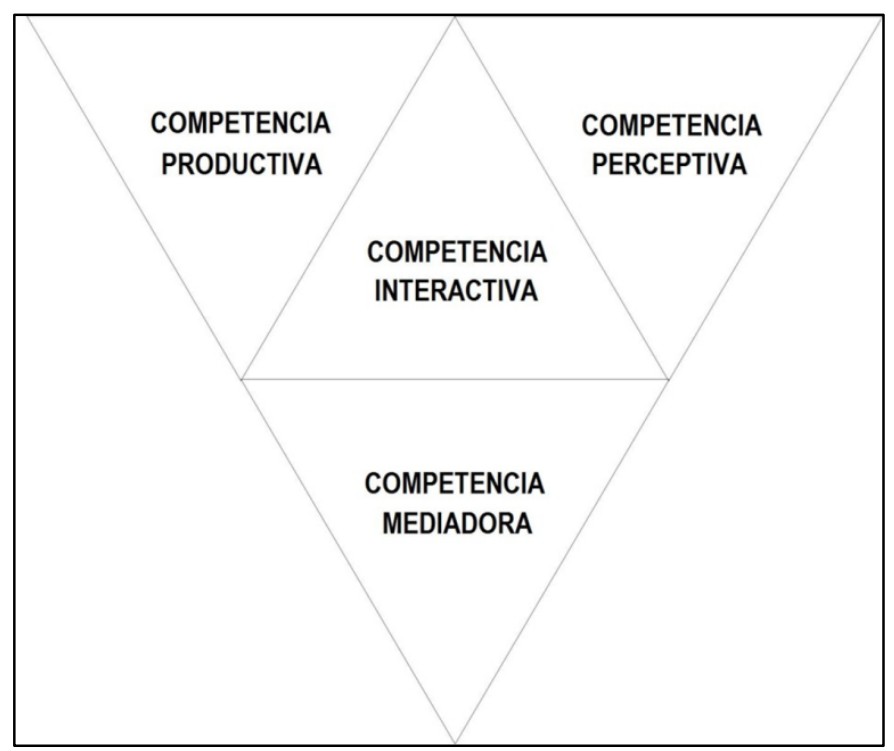

Figura 1. Competencias específicas

Las actividades comunicativas de producción y de percepción son actividades exclusivamente unidireccionales: como dictar una conferencia o asistir a ella; las actividades de mediación y de interacción, en cambio, son dialógicas (bidireccionales y, frecuentemente, multidireccionales), como participar en una conversación. La

\footnotetext{
${ }^{17}$ Sobre las actividades de mediación lingüística, $c f r$. Cantero y de Arriba (2004).
} 
interacción, en cualquier caso, no puede comprenderse como una mera suma de producción más percepción, sino que implica una serie de recursos más complejos, de índole muy diversa al de las actividades unidireccionales.

Además de las competencias específicas, referidas a las actividades comunicativas concretas, cada hablante cuenta con un conjunto de competencias estratégicas que consisten en los recursos, saberes y habilidades, de carácter general, que le permiten afrontar cualquier situación comunicativa:

-la competencia lingüística es la capacidad de conocer y relacionar (paradigmáticamente) las unidades del código,

- la competencia discursiva es la capacidad de relacionar (sintagmáticamente) las unidades del código en situaciones de enunciación efectiva,

-la competencia cultural es la capacidad de manejar los saberes implicados en la comunicación,

- la competencia estratégica es la capacidad de relacionar todas las demás competencias para gestionar las diversas situaciones comunicativas a que se enfrenta el hablante.

También estas competencias se relacionan entre sí con una cierta jerarquía, por cuanto el eje y el motor de todas ellas es la competencia estratégica. Las representamos así (figura 2):

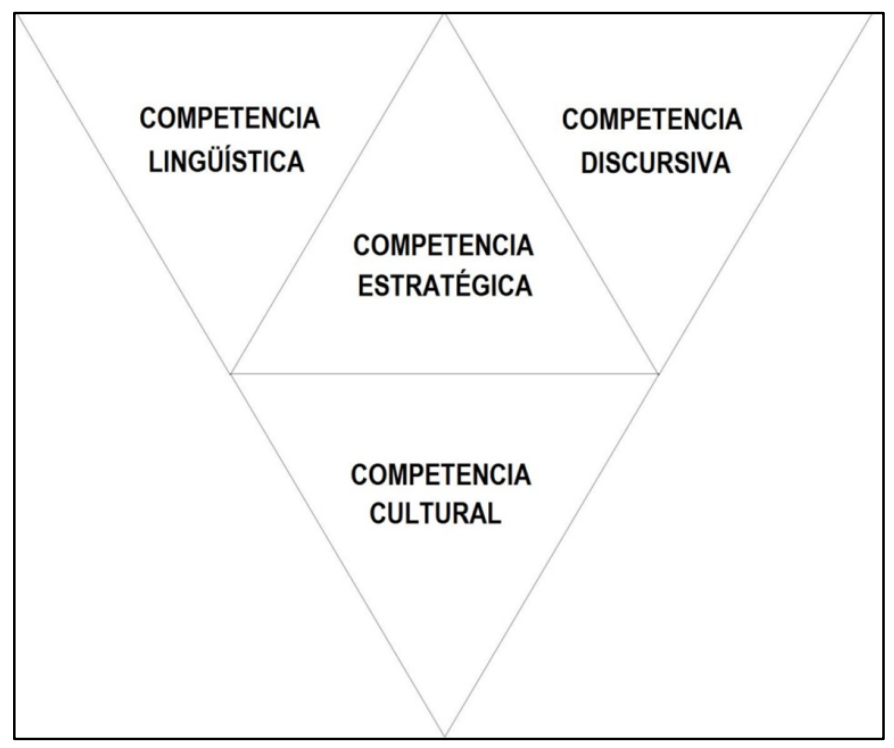

Figura 1. Competencias estratégicas 
La lingüística se ha ocupado, especialmente, de la competencia lingüística y las modernas lingüísticas del texto, análisis del discurso o pragmalingüística de la competencia discursiva. De hecho, para muchos lingüistas los términos «competencia lingüística» $\mathrm{y}$ «competencia comunicativa» aún hoy son sinónimos ${ }^{18}$.

Las competencias específicas y estratégicas se relacionan entre sí con una cierta fractalidad y jerarquía: cada competencia estratégica incluye las distintas competencias específicas. Podemos expresarlo así (figura 3):

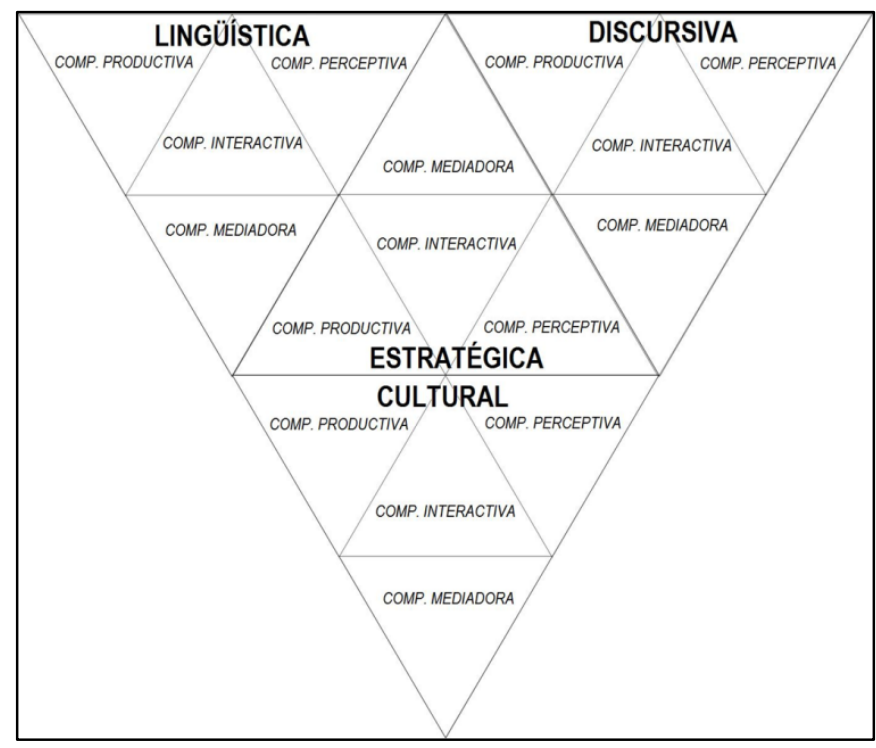

Figura 2. Competencias comunicativas

La competencia comunicativa, por tanto, está constituida por una notable diversidad de subcompetencias, cuyo desarrollo será desigual y dependerá, en todo caso, de la historia de vida del hablante, sus contextos de habla, sus interlocutores, etc.

\subsection{La estrella comunicativa}

La figura 3 sería la representación de las competencias comunicativas implicadas en el uso de un único código de comunicación. Sin embargo, y como vimos, sabemos que los hablantes manejan múltiples códigos alternativos (e incluso simultáneos: como los códigos verbales y no verbales), incluyendo distintos idiomas o variedades de un idioma.

En realidad, todos los hablantes somos, de un modo u otro, multilingües.

Así, la competencia comunicativa de un hablante habría que entenderla, más bien, como una estrella con un número indefinido de puntas, en la que cada punta (o cada

${ }^{18}$ El origen del concepto de «competencia comunicativa» está en la «competencia lingüística» postulada por Chomsky (1965) para referirse a las reglas cuyo conocimiento tácito permitían al hablante generar oraciones gramaticales. Desde el ámbito de la sociolingüística, Hymes (1971) amplió el concepto, añadiéndole una dimensión pragmática, de adecuación al contexto. 
hoja) representaría cada unos de los códigos que conoce o emplea el individuo (figura 4):

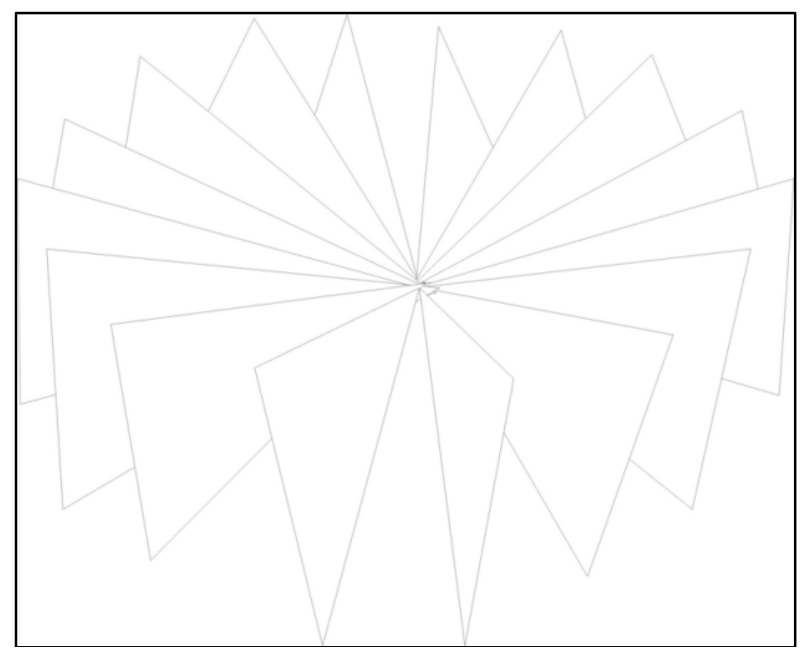

Figura 3. La estrella comunicativa

El eje de la competencia comunicativa (de la «estrella comunicativa») es la competencia estratégica (v. figura 5), común a todos los códigos que maneja el hablante, porque consiste en los recursos o estrategias que pone en juego para relacionar todas las demás subcompetencias.

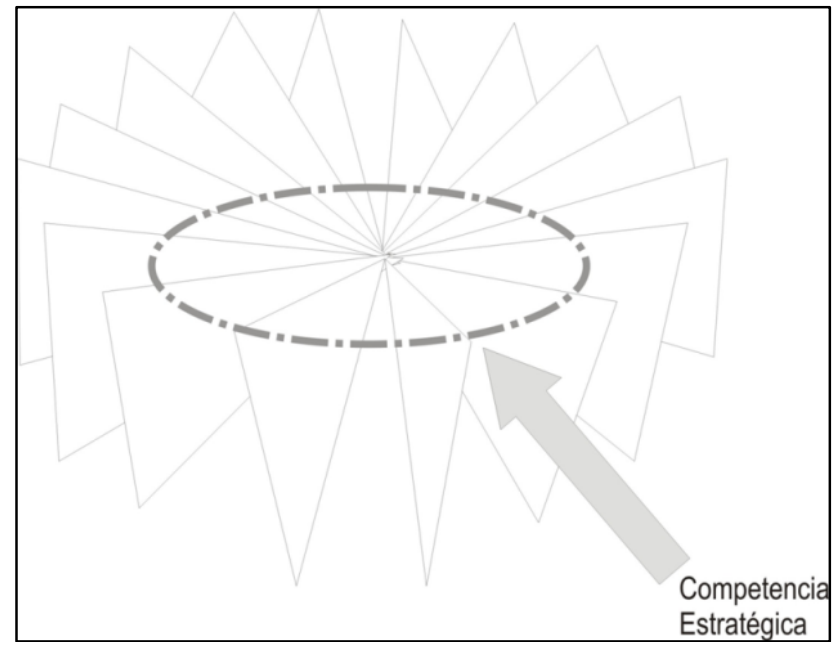

Figura 4 - La competencia estratégica como eje de la competencia comunicativa

La competencia estratégica, así, sería el foco de atención prioritario de la descripción lingüística ${ }^{19}$, por delante de las demás competencias generales, como la

${ }^{19}$ Como lo es de los profesores de lengua (cfr. Cantero, 2014a), por cuanto determina la zona de de desarrollo próximo del hablante (cfr. Vigotsky, 1934). 
lingüística, la discursiva o la cultural, porque en ella se concentra el potencial comunicativo del individuo.

\subsection{Implicaciones teóricas}

Desde esta perspectiva, comprobamos que la fonética y la fonología se han ocupado, tradicionalmente, de una parte muy pequeña de la competencia comunicativa de los hablantes: esencialmente, de la competencia lingüística y, dentro de ella, de la competencia productiva. Una única subcompetencia de las muchas que componen la competencia comunicativa de un solo código: porque, además, solo se han ocupado de un único código (la lengua estándar), de entre todos los códigos que conocen y emplean los hablantes.

La lengua estándar, en realidad, no puede considerarse un lenguaje «natural», sino que ha de ser aprendido en la escuela y, esencialmente, está regulado como lenguaje escrito (eventualmente, también como lenguaje «oral formal» ${ }^{20}$ ).

Un modelo de pronunciación cerrado y unívoco, por tanto, apenas puede dar cuenta de la pronunciación implicada en cualquier proceso de comunicación entre hablantes reales. Solo puede ser un referente teórico, pero no puede describir, ni entender, ni explicar el componente fónico la comunicación.

Si la fonética clásica se ha ocupado, básicamente, de la producción en competencia lingüística, en el análisis del habla espontánea nos encontramos con fenómenos que se corresponden con otras competencias, y con códigos diversos. El análisis acústico de conversaciones auténticas, entonces, podemos suponer que reflejará, de algún modo, lacompetencia interactiva de los participantes, así como su competencia estratégica (su adecuación al contexto y a los interlocutores).

Nuestros resultados del análisis de habla espontánea vendrían a ser como una imagen congelada de un proceso dinámico: diversos sistemas de diversos interlocutores, en plena negociación.

Frente a la creencia de que los hablantes comparten «un mismo» sistema fonológico, la realidad nos enseña que no hay un único sistema fonológico sino múltiples sistemas, que nunca están cerrados sino en constante proceso de construcción y reformulación: múltiples sistemas que han ido elaborándose desde cada subcompetencia de cada código de los diversos códigos que cada hablante pone en juego para llevar adelante sus relaciones.

Para describir, entender, explicar (y luego aplicar) el componente fónico de la comunicación no podemos postular simples sistemas cerrados y unívocos, sino sistemas abiertos, incompletos y dinámicos, que a lo sumo compartirán algunos rasgos comunes, justo los que permitirán a los hablantes negociar sus recursos y ajustarlos con los demás hablantes para, sencillamente, poder entenderse.

20 El lenguaje «oral formal» es exclusivamente unidireccional, frente al lenguaje oral «conversacional». 


\section{FONOLOGÍA DE LA COMPLEJIDAD}

Desde el análisis del habla espontánea, por tanto, es necesario reformular nuestras creencias teóricas y ajustarlas a un nuevo modelo, necesariamente abierto, dinámico, diverso, múltiple, no lineal, emergente: el pensamiento complejo ${ }^{21}$ nos ofrece un paradigma interpretativo y explicativo con el que podríamos afrontar el reto. De hecho, contamos ya con algunas aproximaciones a la fonología desde el pensamiento complejo, como Silverman (2006) o Pellegrino et al. (2009).

\subsection{Fonología de la interacción}

En interacción no hay pronunciaciones unívocas, sino muy variables, como hemos visto: pronunciaciones negociadas.

Los interlocutores negocian los márgenes de dispersión de sus vocales, su timbre, las variantes de cada consonante, el ritmo del habla, la entonación prelingüística (es decir, su acento), el valor de las inflexiones tonales (lingüístico y paralingüístico), así como los códigos que entran en juego en la conversación, el estilo de habla, etc. ${ }^{22}$. Todos los aspectos que forman parte de la fonética del habla son negociados (tácitamente) por los interlocutores.

Los hablantes, en suma, se adaptan al contexto y a sus interlocutores, negocian con ellos y emplean múltiples recursos, códigos alternativos y variantes diversas, con tal de conseguir una comunicación eficaz.

Las distintas variantes de pronunciación de, por ejemplo, la / $\mathrm{j} /$ del castellano ( $v i d$. supra) parece que se solapan en la foto fija que ofrece el análisis acústico de diversas conversaciones, lo cual se nos aparece como un panorama contradictorio. Tales variantes, sin embargo, solo se solapan cuando intentamos creer que los hablantes están manejando «un mismo» código, pero no hay contradicción alguna cuando entendemos que cada hablante ha elaborado un código (diversos códigos) de pronunciación adecuado para esa enunciación, que pone en juego en una situación comunicativa concreta y que negocia con su interlocutor: en la comunicación auténtica, las ambigüedades y las confusiones constituyen la piedra de toque del intercambio, y rara vez persisten después de la negociación implícita a la que llamamos «hablar».

En la comunicación real, se negocian los roles, los estilos, los códigos, los significados, las intenciones $^{23}$; pero también las pronunciaciones, los ritmos, las entonaciones. En eso consiste la comunicación, y por eso no hay comunicación sin negociación.

${ }^{21}$ El pensamiento complejo constituye un nuevo paradigma filosófico, cuyo autor más visible esEdgar Morin. Su obra fundacional fue Morin (1977); otras referencias de interés para comprender el pensamiento complejo son: Morin (1990, 2004, 2007), así como la web <http://www.edgarmorin.org/>. Para una primera aproximación a la complejidad en lingüística, $c f r$. Ballesteros (2011).

22 Vid. en Cantero (2014a) algunos ejemplos de negociación fónica, en cada una de las competencias comunicativas.

${ }^{23}$ Todos aquellos aspectos que estudia el análisis del discurso, el análisis de la conversación y el análisis del lenguaje coloquial. En nuestro idioma, son particularmente relevantes las aportaciones del grupo Val.Es.Co (<www.valesco.es $>)$. 
La negociación continua provoca fenómenos de emergencia: nuestros análisis de habla espontánea describen las pronunciaciones emergentes que aparecen en la conversación. «Emergente» quiere decir: no era previsible ${ }^{24}$. La fonética de lo imprevisto es la fonética de la emergencia.

En interacción, por tanto, no tiene sentido proponer sistemas fonológicos definidos, unívocos, lineales, cerrados, sino rasgos fonéticos permeables, dinámicos, no lineales, que permitan explicar los fenómenos de emergencia en que consiste la fonética del habla espontánea.

\subsection{Sistemas fonológicos dinámicos, no lineales, emergentes}

Entendidos como «sistemas complejos», pues, los sistemas fonológicos deberían ser sistemas dinámicos y no lineales, capaces de dar cuenta de los fenómenos fónicos emergentes:

- Sistemas dinámicos y no «estáticos», porque no es posible, como hemos visto, entender la dimensión fónica de la comunicación oral con referentes teóricos cerrados yunidades estáticas.

En Carré (2009), por ejemplo, se propone un «enfoque dinámico» para entender las características fónicas del habla, a partir de su trabajo sobre el modelado acústico (cfr. Mrayati et al., 1988) y perceptivo de las vocales, aunque «los resultados previos que presentamos aquí sobre las vocales deben extenderse a las consonantes, muchas de las cuales son intrínsecamente dinámicas» (Carré, 2009: 77).

Sobre el enfoque dinámico, dice este autor: «el enfoque dinámico necesita desarrollar nuevas maneras de pensar y nuevas herramientas. Las transiciones formánticas no pueden obtenerse de una mera sucesión de valores estáticos, sino de direcciones y pendientes [...]. El enfoque dinámico no es un enfoque estático que tenga en cuenta parámetros dinámicos, sino que debe ser un enfoque intrísecamente dinámico», y añade: «se requiere un estudio epistemológico de la naturaleza dinámica del habla» (op. cit.: 78), como el que se propone en Carré et al. (2007).

- Sistemas no lineales, multidimensionales, y no sistemas lineales o unívocos: que contemplen la multiplicidad de los códigos orales, la coestructuración de los códigos verbales y no verbales, así como la multidimensionalidad de cada fenómeno fónico.

Muchas de las supuestas ambigüedades que contemplan los estudios lingüísticos solo tienen sentido referidos al análisis del lenguaje escrito, incluyendo el análisis de las transcripciones de textos orales: en la escritura no aparecen los fenómenos fónicos desambiguadores, como la estructuración rítmica y melódica del enunciado, por ejemplo; tampoco en la transcripción (por muy estrecha que sea) aparecen los rasgos de timbre, acento o melodía que pertenecen a niveles de análisis prelingüístico o paralingüístico.

24 Por «emergencia» entendemos la aparición de fenómenos que no son explicables mecánicamente como el producto de sus elementos contituyentes. La «emergencia» es uno de los conceptos clave del pensamiento complejo: $c f r$. una visión de conjunto en Johnson (2001). 
En la comunicación oral, la pronunciación siempre es sincrética, y en ella se reflejan, simultáneamente, todos los rasgos acústicos, tonales y no verbales que informan los distintos niveles de análisis.

En lengua escrita, por ejemplo, la diferencia entre las palabras hola y ola parece evidente, por su ortografía, entendiendo, eso sí, que se trata de dos palabras homófonas; sin embargo, realmente no son (ni han sido nunca) homófonos, porque el nombre ola carece del rasgo de énfasis que sí incorpora el saludo hola: que consiste en una entonación determinada, sin la cual hola se pronunciaría ola ${ }^{25}$. Junto a la entonación enfática, también forma parte de la palabra un gesto con la mano o un gesto facial. Hola requiere, por tanto, la pronunciación de los sonidos ['ola], en efecto, más la pronunciación de una melodía enfática, más la producción de un gesto determinado: todo ello constituye la palabra hola, que (como todas las demás palabras del idioma) no es simplemente lineal, sino multidimensional.

- Sistemas emergentes: cada hablante ha ido elaborando (y sigue haciéndolo a lo largo de su vida) una serie de «sistemas fónicos» borrosos, en cada una de sus múltiples competencias y subcompetencias; y todos ellos son sistemas poco sistemáticos y absolutamente permeables, unos con otros. Permeables, tanto entre los sistemas elaborados para cada actividad comunicativa, como entre los sistemas elaborados en cada uno de los códigos que conoce y emplea dentro de un mismo idioma (variedades dialectales, de registro, etc.), como entre los elaborados en sus distintos idiomas (si emplea regular o esporádicamente algún otro idioma).

Estos «sistemas» asistemáticos, permeables, borrosos... (que tanta perplejidad teórica nos han provocado) no conducen, sin embargo, a ninguna confusión porque no son sistemas cerrados y prêt à porter que el hablante tome y use sin más, sino que son meros principios listos para negociar con un interlocutor. Y ese interlocutor también dispone de sus propios sistemas asistemáticos, permeables, borrosos, listos para ser negociados.

La negociación entre los múltiples sistemas de uno y otro (o varios, cuando la conversación es a tres o más interlocutores) no puede contemplarse ni entenderse como la suma de los sistemas personales de cada uno, sino que constituye una nueva realidad, distinta de los elementos previos a la negociación.

Esa es la pronunciación emergente, y ese es el objeto de estudio en el análisis de habla espontánea.

Esa pronunciación emergente se convierte, en cada intercambio feliz, en fuente y motor de esos sistemas fónicos borrosos y permeables de los interlocutores (la negociación implícita supone un cierto nivel de contagio), que pondrán en juego en su próximo intercambio comunicativo.

\footnotetext{
${ }^{25}$ Sobre los falsos homófonos y falsos homónimos, $c f r$. Cantero (2002): 49-50.
} 


\subsection{Fonología dialógica, autoorganizada, hologramática}

La comunicación es esencialmente dialógica, en sus dos acepciones: es un fenómeno basado en la interacción (el diálogo) y es un fenómeno dialéctico. No es «un hecho», ni una cosa, sino un proceso, una «acción» que pone en relación de manera dialéctica (es decir, de manera dialógica) elementos contrapuestos: diversos hablantes, cada uno con su propio modelo del mundo ${ }^{26} \mathrm{y}$ con sus propias competencias (entre las cuales emergen los sistemas fónicos).

Si las fonologías clásicas son «monológicas» ${ }^{27}$, centradas en la producción (o la percepción) pero no en la interacción, nosotros creemos que una fonología que pretenda entender (y explicar) los sonidos del habla debe incorporar en su ADN la interacción (entre los hablantes, y entre sus sistemas borrosos) como un principio esencial. Mejor que suponer un hablante «ideal», es suponer hablantes que hablan con otros y que negocian y que no son nada ideales, sino concretos e imperfectos, pero perfectamente capaces (esto es, competentes) de regular su comunicación.

Según Silverman (a partir de su estudio sobre el variacionismo), la fonología es un sistema «autoorganizado» y «autosostenido» de convenciones sociales, que evoluciona a lo largo de generaciones de hablantes, producto de «la interacción compleja entre fuerzas articulatorias, acústicas, aerodinámicas, perceptivas, funcionales y sociales» (2006: 216).

Nuestra visión de la fonética como objeto de (continua) negociación entre los interlocutores coincide, esencialmente, con la perspectiva de este autor: la autoorganización de los sistemas fónicos reside en la interacción entre sus elementos y en la interacción con los sistemas de otros hablantes.

Los sistemas fónicos que cada hablante elabora en interacción con otros hablantes y los sistemas emergentes que produce esa interacción, como dijimos, se retroalimentan: desde Vigotsky (1934) sabemos que el habla del niño es producto de su interacción con otros hablantes y que su «monólogo interior», por ejemplo, reproduce los diálogos en que ha participado. Así sigue ocurriendo a lo largo de toda nuestra vida: las interacciones en que participamos condicionan y modifican de algún modo los sistemas que ponemos en juego en la propia interacción ${ }^{28}$.

No hay sistemas ajenos a su uso, ni sistemas establecidos, sino sistemas (borrosos y permeables) que se autorregulan a medida que se emplean (hablar es un juego que se aprende jugando: cfr. Brunner, 1983): sistemas negociados, autoorganizados $\mathrm{y}$ dialógicos.

Nuestra perspectiva sobre la competencia comunicativa implica que cada hablante desarrolla las diversas competencias y subcompetencias de cada uno de sus códigos a lo largo de su vida, en procesos de interacción. El eje de toda la Competencia Comunicativa, como vimos, es la competencia estratégica, que permite relacionar y emplear todos los recursos, saberes y habilidades del hablante (entre los cuales, el

\footnotetext{
${ }^{26}$ Que tampoco son estáticos, sino procesos en construcción: $c f r$. Cantero y de Arriba (1997)

${ }^{27}$ Referidas al «monólogo», es decir, a la comunicación unidireccional.

${ }^{28}$ Como un bucle recursivo: $c f r$. Morin (1990).
} 
principal es su capacidad de negociación) para afrontar una nueva situación comunicativa.

La complejidad de la competencia comunicativa no implica, sin embargo, ninguna dispersión, ninguna contradicción: la competencia estratégica del individuo engarza todas las demás competencias, las coordina y las cohesiona. Y en cada nuevo proceso comunicativo entra en juego toda la competencia comunicativa en su conjunto, por concreto que sea el intercambio: la competencia comunicativa diríamos que es como un holograma, en el que cada parte refleja, de alguna manera, la totalidad de competencias, y el todo se refleja en cada parte.

La pronunciación puesta en juego en una interacción determinada se negocia con el interlocutor, como hemos dicho, y en esa negociación participan todas las estrategias del hablante (todas las estrategias de todas las competencias de cada código que el hablante maneja), y ese principio hologramático de la pronunciación es el que permite la negociación entre los interlocutores, que no están limitados por un sistema cerrado y unívoco (como creíamos desde perspectivas tradicionales) sino que se mueven con libertad por la totalidad de sus competencias.

La fonología de la complejidad, por tanto, entendemos que ha de atender a los sistemas dinámicos, no lineales, emergentes, dialógicos, autoorganizados $\mathrm{y}$ hologramáticos que el hablante pone en juego en la comunicación y a los que nos acercamos, imperfectamente y llenos de perplejidades, desde el análisis del habla espontánea.

\section{REFERENCIAS BIBLIOGRÁFICAS}

Alfonso LozAno, Raúl (2010): El vocalismo del español en habla espontánea, tesis doctoral inédita, Barcelona, Universitat de Barcelona.

ALFONSO LOZANO, Raúl (2014): «El vocalismo del español en habla espontánea», Phonica, vol. 9-10 [en $<$ http://revistes.ub.edu/index.php/phonica/article/view/10943 $>$.

ANDRÉS EDO, Belén (2014): «Análisis acústico de los sonidos laterales en el habla espontánea del español», Phonica, vol. 9-10 [en línea]: $<$ http://revistes.ub.edu/index.php/phonica/article/view/10944 $>$.

BARTOLÍ, Marta (2005): «La pronunciación en la clase de lenguas extranjeras», Phonica, vol. 1 [en línea]: $<$ http://revistes.ub.edu/index.php/phonica/article/view/5565/7353 $>$.

BARTOLÍ, Marta (2012): La pronunciación por tareas en la clase de ELE, tesis doctoral, Barcelona, Universitat de Barcelona [en línea]: $<$ http://tdx.cat/handle/10803/134881 $>$.

BARtolí, Marta (2014): «La pronunciación por tareas en la clase de ELE», Phonica, vol. 9-10

[en línea]: <http://revistes.ub.edu/index.php/phonica/article/view/10976>. 
BALlesteros, Mapi (2011): «Pensamiento complejo y estudio de la comunicación», Phonica, vol. 7

[en línea]: $<$ http://revistes.ub.edu/index.php/phonica/article/view/5604 $>$.

Ballesteros, Mapi, Miguel Mateo y Francisco José Cantero Serena (2010): «Corpus oral para el análisis melódico de las variedades del español», Actas del XXXIX Simposio Internacional de la SEL. Santiago de Compostela: Universidad de Santiago de Compostela.

BRUNNER, Jerome (1983): Child's Talk: Learning to Use Language, Nueva YorkLondres, W. W. Norton. Trad. española: (1986): El habla del niño, Barcelona, Paidós, $<$ http://dx.doi.org/10.1177/026565908500100113>.

Bunge, Mario (1969): La investigación cientifica, Barcelona, Ariel (sucesivas reediciones en México, Siglo XXI).

Bunge, Mario (1983): Lingüistica y filosofía, Barcelona, Ariel.

CAntero Serena, Francisco José (1998): «Conceptos clave en lengua oral», en Mendoza, Antonio, coord., Conceptos clave en didáctica de la lengua y la literatura, Barcelona, Horsori, 141-153.

Cantero Serena, Francisco José (2002): Teoría y análisis de la entonación, Barcelona, Edicions de la Universitat de Barcelona.

CANTERo Serena, Francisco José (2003): «Fonética y didáctica de la pronunciación», en Mendoza, Antonio, coord., Didáctica de la Lengua y la Literatura, Madrid, Pearson, 545-572.

Cantero Serena, Francisco José (2004): «Comunicació i veu», Articles, vol. 32, 8-24.

CANTERo Serena, Francisco José (2008): «Complejidad y competencia comunicativa», Revista Horizontes de Lingüística Aplicada, vol. 7, núm. 1, 71-87 [en línea]: $<$ http://seer.bce.unb.br/index.php/horizontesla/article/view/2994/2596>.

CANTERo Serena, Francisco José (2014a): «Adquisición de competencias fónicas», en Congosto, Yolanda et al., eds., Fonética Experimental, Educación Superior e Investigación, vol. II, Madrid, Arco-Libros, 29-55.

CANTERo Serena, Francisco José (2014b): «Códigos de la puntuación y entonación emocional», en Díaz Galán, Ana et al., eds., Comunicación, Cognición, Cibernétic@. Actas del 31. ${ }^{\text {r }}$ Congreso Internacional de la Asociación Española de Lingüistica Aplicada, Tenerife, Universidad de La Laguna, 618-629.

Cantero Serena, Francisco José y Clara DE Arriba (1996): «El cambio de código: contextos, tipos y funciones», en Otal, José Luis, Inmaculada Fortanet y Victoria Codina, eds., Estudios de Lingüística Aplicada, Castellón, Publicacions de la Universitat Jaume I.

CANTERo Serena, Francisco José y Clara DE Arriba (2004): «La mediación lingüística en la enseñanza de lenguas», Didáctica (Lengua y Literatura), vol. 16, 9-21 [en línea]:

$<$ http://www.ucm.es/BUCM/revistas/edu/11300531/articulos/DIDA0404110009A.PDF>.

CANTERo Serena, Francisco José y José DE ARribA (1997): Psicolingüistica del discurso, Barcelona, Octaedro.

Cantero Serena, Francisco José y Miguel Mateo (2011): «Análisis melódico del habla: complejidad y entonación en el discurso», Oralia, vol. 14. 
Cantero Serena, Francisco José, Raúl Alfonso, Marta Bartolí, Anna Corrales y Maribel Vidal (2005): «Rasgos melódicos de énfasis en español», Phonica, vol. 1 [en línea]: $<$ http://revistes.ub.edu/index.php/phonica/article/view/5571>.

CARRÉ, René (2009): «Signal dynamics in the production and perception of vowels», en Pellegrino, Françoise et al., eds. (2009), Approaches to phonological complexity, Berlín-Nueva York, Mouton de Gruyter, 59-81, $<$ http://dx.doi.org/10.1515/9783110223958.59>.

CARré, René, François Pellegrino y Pierre DiVenyi (2007): «Speech dynamics: epistemological aspects», Proceedings of the ICPhSSaarbrücken, 569-572.

CHOMsky, Noam (1965): Aspects of the Theory of Syntax, Cambridge, MIT Press.

COUNCIL FOR CUlTURAL COOPERATION (2001): Common European Framework of Reference for Languages: Learning, Teaching, Assessment.Strasbourg: Council of Europe. (2002): Marco común europeo de referencia para las lenguas: aprendizaje, enseñanza, evaluación, Instituto Cervantes [en línea]: $<$ http://cvc.cervantes.es/obref/marco $>$.

DEvís, Empar (2011): «La entonación de (des)cortesía en el español coloquial», Phonica, vol. 7 , [en línea]: $<$ http://revistes.ub.edu/index.php/phonica/article/view/5606>.

Devís, Empar y Francisco José CANTERO (2014). «The intonation of mitigating politeness in Catalan», Journal of Politeness Research, vol. 10, núm. 1, 127-149, $<$ http://dx.doi.org/10.1515/pr-2014-0006>.

FónAgy, I. (1983): La vive voix, París, Payot.

FONT-RotCHÉS, Dolors (2011): «Ėmfasis bàsics de l'entonació en els contorns del català central», en Lloret, Maria-Rosa y Clàudia Pons, coords., Noves aproximacions a la fonologia $i$ morfologia del català, Alacant, Institut Universitari de Filologia Valenciana, 193-216.

FONT-RotCHÉS, Dolors (2014): «Rasgos melódicos de la entonación paralingüística de foco», en Díaz Galán, Ana et al., eds., Comunicación, Cognición, Cibernétic@. Actas del 31. ${ }^{\text {er }}$ Congreso Internacional de la Asociación Española de Lingüística Aplicada, Tenerife, Universidad de La Laguna, 686-701.

Giralt LoRenz, Marta (2012): El enfoque oral en la iniciación de la enseñanza/aprendizaje y adquisición de la pronunciación del español como lengua extranjera, tesis doctoral, Barcelona, Universitat de Barcelona [en línea]: $<$ http://www.tdx.cat/handle/10803/94136 $>$.

GiRAlt LORENZ, Marta (2014): «Una aproximación al enfoque oral para la enseñanza y aprendizaje de la pronunciación del E/LE», Phonica, vol. 9-10 [en línea]: $<$ http://revistes.ub.edu/index.php/phonica/article/view/10978 $>$.

Guaus, Roger, Jaume Oliver, Francesc GudAYOL y Josep MARTí (1997): «Síntesis de voz utilizando difonemas: uniones entre vocales», Procesamiento del lenguaje natural, vol. 21, 69-74, [en línea]: <http://hdl.handle.net/10045/2075>.

GumPerZ, John J. (1982): Discourse Strategies, Cambridge, Cambridge University Press, $<$ http://dx.doi.org/10.1017/cbo9780511611834>.

Heller, Monica (ed.) (1988): Code-switching, Berlín, Mouton de Gruyter, $<\underline{\text { http://dx.doi.org/10.1515/9783110849615 }>\text {. }}$ 
HyMES, Dell Hathaway (1971): «On communicative competence», en Pride, John B. y Janet Holmes, eds., (1972): Sociolinguistics: Selected readings, Londres, Penguin Education.

Johnson, Steven (2001): Emergence: The Connected Lives of Ants, Brains, Cities and Software, Londres, The Penguin Press. Trad. española (2001): Sistemas emergentes. $O$ qué tienen en común hormigas, neuronas, ciudades y software, Madrid, Fondo de Cultura Económica.

MORIN, Edgar (1977): La méthode. 1. La nature de la nature, París, Seuil. Trad. española (1980), El método I. La naturaleza de la naturaleza, Madrid, Cátedra.

MoRIN, Edgar (1990): Introduction à la penséecomplexe, París, ESF éd. Trad. española (2004): Introducción al pensamiento complejo, México, D. F., Editorial Gedisa.

Morin, Edgar (2004): «La epistemología de la complejidad», Gazeta de Antropología, vol. 20 [en línea]: <http://hdl.handle.net/10481/7253>. Páginas 43-77 de Morin, Edgar (1999): L'intelligence de la complexité, París, L'Harmattan.

MORIN, Edgar (2007): On complexity. Cresskill, Hampton Press.

MrayATI, Mohammed, René CARRÉ y Bernard GUÉRIN (1988): «Distinctive regions and models: A new theory of specch production», Speech Communication, vol. 7, 257-286, < http://dx.doi.org/10.1016/0167-6393(88)90073-8>.

Navarro Tomás, Tomás (1918): Manual de pronunciación española, Madrid, Centro de Estudios Históricos. Desde la 4. a edición (1932), corregida y aumentada, Madrid, CSIC $\left(1989^{23}\right)$.

ORTIZ DE PINEDO, Núria (2012): «Las vibrantes del español en habla espontánea», en $\begin{array}{llll}\text { Phonica, } & \text { vol. } & 8 & \text { [en }\end{array}$ $<$ http://revistes.ub.edu/index.php/phonica/article/view/10189>.

ORTIZ DE PINEDO, Núria (2014): «Análisis acústico de la vibrantes del español en habla espontánea», Phonica, vol. 9-10 [en línea]:

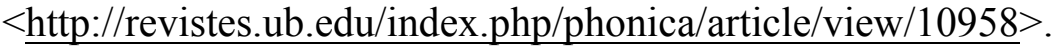

Pellegrino, François, Egidio Marsico, Ioana Chitoran y Christophe Coupé (eds.) (2009): Approaches to phonological complexity, Berlín-Nueva York, Mouton de

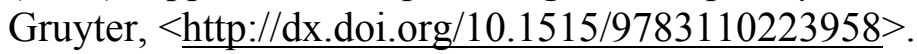

RiUs-EsCUdÉ, Agnès (2011): Les vocals mitjanes anteriors $i$ posteriors del català central en parla espontània. Treball d'investigació del Màster de Recerca en Didàctica de la Llengua i la Literatura, Barcelona, Universitat de Barcelona [en línea]: $<$ http://hdl.handle.net/2445/20604>.

Rius-Escudé, Agnès (2014): «Tretsacústics de les vocals del català central», Phonica, vol. 9-10 [en línea]: <http://revistes.ub.edu/index.php/phonica/article/view/10959>.

RiUs-Escudé, Agnès y Francina TORRAs (2014): «Dispersión e influencia del punto de articulación del sonido adyacente en las vocales medias posteriores en catalán», Congosto, Yolanda et al., eds., Fonética Experimental, Educación Superior e Investigación, vol. I, Madrid, Arco-Libros, 419-436.

RIUs-EsCudÉ, Agnès y Francina TORRAs (2015): «Anàlisi acústica contrastiva de les vocals mitjanes posteriors del català $i$ del castellà en parla espontània», Actes del XVIè Col-loqui Internacional de Llengua $i$ Literatura Catalanes, vol. II, Barcelona, Publicacions de l'Abadia de Montserrat, 283-296. 
Rousselot, Jean-Pierre (1908): Principes de phonétique expérimentale (tomo I, $1897-$ 1901; tomo II, 1901-1908), París-Leipzig, Welter.

RousSELOT, Jean-Pierre y Fauste LAClOTTE (1902): Précis de prononciation française, París-Leipzig, Welter.

Silverman, Daniel (2006): A critical introduction to phonology (of sound, mind aln body), Londres-Nueva York, Continuum.

SOla PRADO, Alicia (2011): "Las aproximantes $[\beta, \delta, \gamma]$ del español en habla espontánea», Phonica, vol. 7 [en línea]:

$<$ http://revistes.ub.edu/index.php/phonica/article/view/5609>.

Sola PRADO, Alicia (2014a): «Estudio sobre las aproximantes [ $[3, \partial, \gamma]$ del español en habla espontánea», Phonica, vol. 9-10 [en línea]:

$<$ http://revistes.ub.edu/index.php/phonica/article/view/10960>.

Sola PRADO, Alicia (2014b): «Caracterización acústica de las aproximantes [ $[\beta, \partial, \gamma]$ del español en habla espontánea», en Congosto, Yolanda et al., eds., Fonética Experimental, Educación Superior e Investigación, vol. I, Madrid, Arco-Libros, 436-464.

TORREgrosa AzOR, José (2006): «Análisis multisistémico de la comunicación humana», Phonica, vol. 2 [en línea]: $<\underline{\text { http://revistes.ub.edu/index.php/phonica/article/view/5650 }>\text {. }}$

TORREgrosa AzOR, José (2011): «Análisis multisistémico de las partículas modales del alemán», Biblioteca Phonica, vol. 13-14 [en línea]: $<\underline{\text { http://revistes.ub.edu/index.php/phonica/article/view/11039> }}$.

TORREGROSA AZOR, José (2011): «Interacció de codisorals en parla espontània: el cas de les partículesmodals de l'alemany», Phonica, vol. 9-10 [en línea]:

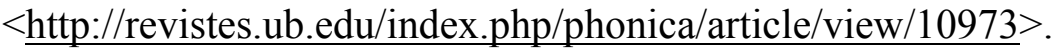

Vigotsky, Lev S. (1934): Myshlenie i rech': Psikhologicheskieissledovaniya. Moscú y Leningrado: GosudarstvennoneSotsial-no-EkonomicheskoeIzdatel'stvo. Trad. española (1977): Pensamiento y lenguaje, Buenos Aires, La Pléyade. (Nueva edición, a cargo de A. Kozulin, en Barcelona, Paidós, 1995). 